\title{
A VARIANCE REDUCTION TECHNIQUE USING A QUANTIZED BROWNIAN MOTION AS A CONTROL VARIATE
}

\author{
A. LEJAY AND V. REUTENAUER
}

\begin{abstract}
This article presents a new variance reduction technique for diffusion processes where a control variate is constructed using a quantization of the coefficients of the Karhunen-Loève decomposition of the underlying Brownian motion. This method may be indeed used for other Gaussian processes.
\end{abstract}

\section{INTRODUCTION}

This article aims to present a new variance reduction technique for Monte Carlo simulations for computing expectations of quantities related to Brownian motion or solutions of stochastic differential equations (SDE). Monte Carlo methods are sometimes the only tool available to perform some simulations and are in general naturally and simply implemented from the model. In mathematical finance, it is customary to use SDE to model the evolution of stocks prices and rates, and except for some simple options, analytical or semi-analytical formulae are not available. Thus, the quantities to compute are obtained by numerically averaging a large number of realizations of stock prices or rates. However, the precision of the Monte Carlo method is limited by the variance of the option price, which is in general not know. A large number of methods, called variance reduction techniques have been proposed to obtain better estimators and to reduce the computational time. There is no need to underline the practical importance of such techniques (see for example the books $[8,10]$ ) which are still an active subject of research. Our scheme relies on a control variate constructed using quantization of some Gaussian random variables.

Quantization techniques consist in replacing a random variable $X$ by a discrete one $\widehat{X}$ that takes its values in a finite set of points $\left\{x_{1}, \ldots, x_{k}\right\}$, and which is chosen to be optimal, say for example such that $\mathbb{E}\left[|X-\widehat{X}|^{2}\right]$ is minimal. If one knows $p_{i}=\mathbb{P}\left[\widehat{X}=x_{i}\right]$, then an approximation of $\mathbb{E}[\phi(X)]$ is easily computed by $\mathbb{E}[\phi(\widehat{X})]=\sum_{i=1}^{k} p_{i} \phi\left(x_{i}\right)$.

Date: June 5, 2009.

1991 Mathematics Subject Classification. Primary 60H35 ; Secondary 60H10, $65 \mathrm{C} 30$.

Key words and phrases. Monte Carlo simulation for diffusion processes, variance reduction, control variate, Karhunen-Loève decomposition, least squares method. 
Initially, quantization techniques have been developed in the fields of signal theory. Recently, several applications to efficient simulation of diffusion processes and Monte Carlo methods have been proposed (see for example $[1,2,6,18,24]$ and the survey articles $[21,25])$. A mathematical treatment of this theory can be found in [11].

Let $B$ be a Brownian motion and $\Phi$ be a measurable function on the space $\mathcal{C}([0, T] ; \mathbb{R})$ of continuous functions from $[0, T]$ to $\mathbb{R}$. We are interested in computing

$$
J=\mathbb{E}[\Phi(B)] .
$$

Let us note that if $B$ is replaced by the solution $X$ to some strong solution of SDE, then there exists a measurable function $\Psi$ such that $X=\Psi(B)$ (This is detailed in Appendix A). As we need to perform simulations on the underlying Brownian motion to work in a Gaussian framework, we may consider without loss of generality expressions of type (1).

The classical Monte Carlo estimator $J^{N}$ for $J$ in (1) consists in simulating $N$ independent copies $B^{(1)}, \ldots, B^{(N)}$ of the Brownian motion, and to compute

$$
J^{N}=\frac{1}{N} \sum_{i=1}^{N} \Phi\left(B^{(i)}\right) .
$$

The variance of $J^{N}$ is equal to $\operatorname{Var}(\Phi(B)) / N$, and depends on the function $\Phi$. Among the possible approaches to construct a new estimator $\widehat{J}^{N}$ that converges to $J$ with a smaller variance, one consists in constructing a random variable $\widehat{B}$ that is close to $B$ - when $\Phi$ is continuous - and such that $\mathbb{E}[\Phi(\widehat{B})]$ can be easily be computed. Then, set

$$
\widehat{J}^{N}=\frac{1}{N} \sum_{i=1}^{N}\left(\Phi\left(B^{(i)}\right)+\beta\left(\Phi\left(\widehat{B}^{(i)}\right)-\mathbb{E}[\Phi(\widehat{B})]\right)\right),
$$

where $\widehat{B}^{(i)}$ is a copie of $\widehat{B}$ associated to $B^{(i)}$. The constant $\beta$ may be taken equal to 1 , or one can choose an optimal constant

$$
\beta=\operatorname{Cov}(\Phi(B), \Phi(\widehat{B})) / \operatorname{Var}(\Phi(\widehat{B})),
$$

that can be approximated using for example some schemes presented in $[8,30] \ldots$ In order to simplify our presentation, we set $\beta=1$.

In our case, we are willing to find a finite number of trajectories that "summarize" the behavior of the Brownian motion. A possibility consists in using a finite number of $\left(t_{0}, t_{1}, \ldots, t_{d}\right)$ of the Brownian motion and to quantize the vector of increments $\left(B_{t_{1}}-B_{t_{0}}, \ldots, B_{t_{d}}-B_{t_{d}-1}\right)$ and then to use linear interpolations to link the points. Another possibility consists in using for $\widehat{B}$ a quantization of the truncated version 
of the Karhunen-Loève decomposition, that is

$$
\widehat{B}_{t}=\sum_{i=1}^{m} \sqrt{\lambda_{k}} \widehat{\xi}_{i} f_{k}(t) \text { for } t \in[0, T]
$$

where the $f_{k}$ 's are $d$ elements of a basis of $\mathrm{L}^{2}([0, T] ; \mathbb{R})$, the $\lambda_{k}$ 's are appropriate scalars, and $\left(\widehat{\xi}_{1}, \ldots, \widehat{\xi}_{m}\right)$ is a quantization of a Gaussian vector $\left(\xi_{1}, \ldots, \xi_{m}\right)$. This means that $\left(\widehat{\xi}_{1}, \ldots, \widehat{\xi}_{m}\right)$ takes it values in a finite set of $M$ points that have been optimized in some way. This quantization is called a functional quantization and the feature of this approach is that the truncation number $m$ can be rather small $(m \leq 10$ for example) to properly catch the global behavior of a trajectory.

The functional quantization has been proposed and studied by G. Pagès et al. as a way to compute quickly an approximation of $\mathbb{E}[\Phi(B)]$ with the expression $\mathbb{E}[\Phi(\widehat{B})]$ (see $[18,26]$ ). He also proposed to use functional quantization as a control variate by simulating $B$ with a truncated Karhunen-Loève decomposition.

Here, our approach is a bit different since we are not bound to replace the Brownian motion by its truncated Karhunen-Loève decomposition. Using a least squares estimator, we construct $\widehat{B}$ from $\left(B_{t_{1}}, \ldots, B_{t_{d}}\right)$, where $\left(t_{1}, \ldots, t_{d}\right)$ are fixed times.

This is important, especially when diffusion processes are simulated, since one can choose the most convenient way to do so and only record the vector of marginals of the underlying Brownian motion.

Note that in our algorithm, the Brownian motion may be replaced by any other Gaussian process, such as Ornstein-Uhlenbeck processes or Brownian bridge.

\section{THE ALGORITHM}

2.1. The idea. The idea behind the algorithm is the following: we fix some integers $d$ and $m \leq d$, some times $0 \leq t_{1}<\cdots \leq t_{d}$, as well as an orthonormal basis $\left\{f_{i}\right\}$ of $\mathrm{L}^{2}([0, T] ; \mathbb{R})$. We compute $\lambda_{k}=\operatorname{Var}\left(\int_{0}^{T} f_{i}(s) B(s) \mathrm{d} s\right)$. Given a realization $\boldsymbol{\beta}=\left(\beta_{1}, \ldots, \beta_{d}\right)$ of $\left(B_{t_{1}}, \ldots, B_{t_{d}}\right)$, we define $\boldsymbol{\xi}=\left(\xi_{1}, \ldots, \xi_{m}\right)$ as

$$
\boldsymbol{\xi}=\operatorname{argmin}_{\left(\zeta_{1}, \ldots, \zeta_{m}\right) \in \mathbb{R}^{m}} \sum_{i=1}^{d}\left(\sum_{j=1}^{m} \zeta_{j} \sqrt{\lambda_{j}} f_{j}\left(t_{i}\right)-\beta_{t_{i}}\right)^{2},
$$

and choose a quantization $\widehat{\boldsymbol{\xi}}$ of this vector. Note that there are several ways to do so: one can compute the optimal quantization of the vector $\boldsymbol{\xi}$, or a product quantization (i.e. each component is quantized independently), or any mixing of the two approaches [23].

We then use $\widehat{B}$ defined by (3) as a control variate. Since we use a quantization of $\boldsymbol{\xi}, \widehat{\boldsymbol{\xi}}$ may take $Q$ different values for some fixed $Q$, 
so that $\widehat{B}$ has $Q$ trajectories, and $\Phi(\widehat{B})$ as well as $\mathbb{E}[\Phi(\widehat{B})]$ can be computed prior to any simulation. For $\mathbb{E}[\Phi(\widehat{B})]$, one needs to know all the probabilities $p^{(i)}=\mathbb{P}\left[\widehat{\boldsymbol{\xi}}=\left(x_{1}^{(i)}, \ldots, x_{m}^{(i)}\right)\right]$ for the $Q$ possible points $\left\{\left(x_{1}^{(i)}, \ldots, x_{m}^{(i)}\right)\right\}_{i=1, \ldots, Q}$ of the quantized vector.

2.2. Preliminary computations. Let $M$ be the $d \times m$-matrix defined by $M_{i, j}=\sqrt{\lambda}_{j} f_{j}\left(t_{i}\right)$. We assume:

(H) The rank of $M$ is $m$.

Solving the linear least squares is then equivalent to solve $M^{T} \boldsymbol{\xi}=\boldsymbol{\beta}$. As $M$ is not necessarily a square matrix, one can indeed equivalently solve

$$
A \boldsymbol{\xi}=M \boldsymbol{\beta} \text { with } A=M M^{T} .
$$

The matrix $A$ is a $m \times m$-symmetric matrix which has rank $m$ under Hypothesis $(\mathrm{H})$ and this is invertible and then $\boldsymbol{\xi}=A^{-1} M \boldsymbol{\beta}$.

The Gaussian vector $\boldsymbol{\beta}$ may be defined as $\boldsymbol{\beta}=N \Delta \mathbf{G}$ where $\mathbf{G}$ is a vector of independent Gaussian random variables with mean 0 and variance $1, N$ is the $d \times d$-matrix defined by $N_{i, j}=1$ is $i \leq j$ and $N_{i, j}=0$ otherwise, and $\Delta$ is the diagonal matrix with $\sqrt{t_{1}}, \sqrt{t_{2}-t_{1}}, \ldots, \sqrt{t_{d}-t_{d-1}}$ as diagonal terms. Hence, we deduce that

$$
\operatorname{Cov}(\boldsymbol{\xi})=\left(A^{-1} M N \Delta\right)\left(A^{-1} M N \Delta\right)^{T} .
$$

In addition, there exists a unique symmetric $d \times d$-matrix $\Gamma$ such that $\operatorname{Cov}(\boldsymbol{\xi})=\Gamma^{2}$, and then $\boldsymbol{\alpha}=\Gamma^{-1} \boldsymbol{\xi} \sim \mathcal{N}\left(0, \operatorname{Id}_{m \times m}\right)$ is a $m$-vector of independent Gaussian random variables.

2.3. The algorithm. The algorithm is then the following.

(1) Choose a number $d$ of marginals to use and a number $m \leq d$ of coefficients, as well as the times $0<t_{1}<\ldots<t_{d} \leq T$.

(2) Choose an orthonormal basis $\left\{f_{j}\right\}_{j \geq 1}$ of $\mathrm{L}^{2}([0, T] ; \overline{\mathbb{R}})$ and compute the corresponding weights $\lambda_{k}$ in the Karhunen-Loève decomposition of the Brownian motion. Compute the matrices $\Gamma$, $\Gamma^{-1}$ and $R=\Gamma^{-1} A^{-1} M$.

(3) Choose a quantization of $m$-dimensional vectors $\boldsymbol{\alpha} \sim \mathcal{N}\left(0, \operatorname{Id}_{m \times m}\right)$, that is a discrete set $\left\{\widehat{\boldsymbol{\alpha}}^{(i)}\right\}_{i=1, \ldots, M}$ called the code book and a "projection" $\pi$ which associates to a realization of $\boldsymbol{\alpha}$ an element of the code book. Compute $p^{(i)}=\mathbb{P}\left[\pi(\boldsymbol{\alpha})=\widehat{\boldsymbol{\alpha}}^{(i)}\right], \phi^{(i)}=\Phi\left(\widehat{B}^{(i)}\right)$ where

$$
\widehat{B}_{t}^{(i)}=\sum_{j=1}^{m} \widehat{\xi}_{j}^{(i)} \sqrt{\lambda_{j}} f_{j}(t) \text { with } \widehat{\boldsymbol{\xi}}^{(i)}=\Gamma \widehat{\boldsymbol{\alpha}}^{(i)},
$$

and $\widehat{J}=\sum \phi^{(i)} p^{(i)}$

(4) Set $J \leftarrow 0$.

(5) For $i$ from 1 to $N$ do 
(a) Simulate a realization $B(\omega)$ of the Brownian motion $B$, record $\boldsymbol{\beta}=\left(B_{t_{1}}(\omega), \ldots, B_{t_{d}}(\omega)\right)$ and compute $\phi=\Phi(B(\omega))$.

(b) Compute $\boldsymbol{\alpha}=R \boldsymbol{\beta}$.

(c) Find the index $i$ such that $\widehat{\boldsymbol{\alpha}}^{(i)}=\pi(\boldsymbol{\alpha})$.

(d) Set $J \leftarrow J+\phi-\phi^{(i)}$.

(6) Return $N^{-1}(J+\widehat{J})$, which is an estimator of $\mathbb{E}[\Phi(B)]$.

Remark 1. For the Brownian motion, there is a natural choice of a basis $\left\{f_{j}\right\}_{j \geq 1}$, which is the set of eigenfunctions of the covariance operator $f \in \mathcal{C}([0, T] ; \mathbb{R}) \mapsto\left(t \mapsto \int_{0}^{+\infty}(s \wedge t) f(s) \mathrm{d} s\right)$ of the Brownian motion. In this case,

$$
\lambda_{j}=\left(\frac{T}{\pi\left(j-\frac{1}{2}\right)}\right)^{2} \text { and } f_{j}=\sqrt{\frac{2}{T}} \sin \left(\pi\left(j-\frac{1}{2}\right) \frac{t}{T}\right)
$$

for $j \geq 1$. Let us note that the algorithm may be applied to any Gaussian process. However, it in not always so easy to get the eigenfunctions of the corresponding covariance operator (see [24] for example).

Remark 2. In this algorithm, we have chosen to use the quantization of Gaussian random vectors with covariance matrix equal to identity. With slight changes in the algorithm, we can either quantize the vector $\Delta^{-1} N^{-1} \boldsymbol{\beta} \sim \mathcal{N}\left(0, \operatorname{Id}_{d \times d}\right)$ of size $d \geq m$ or the vector $\boldsymbol{\xi} \sim(0, \operatorname{Cov}(\boldsymbol{\xi}))$, which have covariances matrices different from identity. Our choice is justified by the numerical cost of the quantization procedure and the need for vectors with sizes as small as possible. Quantizations of $m$ vectors with distribution $\mathcal{N}\left(0, \operatorname{Id}_{m \times m}\right)$ may be prepared prior to any simulations [23]. In addition, tabulated values can be found in the WEB site [22], thanks to the works of G. Pagès and J. Printems.

\section{Numerical TESTS}

We have performed numerical tests with 3 classical models in finance: the Black \& Scholes model

$$
X_{t}=X_{0}+\int_{0}^{t} \sigma X_{s} \mathrm{~d} B_{s}^{1}+\int_{0}^{t} b\left(X_{s}\right) \mathrm{d} s=X_{0} \exp \left(\sigma B_{t}+\left(\mu-\frac{1}{2} \sigma^{2}\right) t\right),
$$

the Cox-Ingersoll-Ross (CIR) model

$$
X_{t}=X_{0}+\int_{0}^{t} k\left(a-X_{s}\right) d s+\sigma \int_{0}^{t} \sqrt{X_{s}} d B_{s}^{1}
$$

and the Heston model

$$
\left\{\begin{array}{l}
V_{t}=V_{0}+\int_{0}^{t} k\left(a-V_{s}\right) d s+\sigma \int_{0}^{t} \sqrt{V_{s}} d B_{s}^{1} \\
X_{t}=X_{0}+\int_{0}^{t} \mu X_{s} d s+\int_{0}^{t} \sqrt{V_{s}} X_{s} d B_{s}^{2} \\
\left\langle B^{1}, B^{2}\right\rangle_{t}=\rho t
\end{array}\right.
$$


where $B^{1}$ and $B^{2}$ are two Brownian motions. The marginals $\left(B_{t_{1}}, \ldots, B_{t_{d}}\right)$ are taken at times $t_{i}=T / 10, i=1, \ldots, 10$.

For this, we have compared three estimators $v, v^{\prime}$ and $v_{\beta}^{\prime}$ of the empirical variance of $\Phi\left(X_{T}\right)$ associated to the three empirical means

$$
\begin{aligned}
\mu & =\frac{1}{N} \sum_{i=1}^{N} \Phi\left(X^{(i)}\right), \\
\mu^{\prime} & =\frac{1}{N} \sum_{i=1}^{N}\left(\Phi\left(X^{(i)}\right)-\left(\Phi\left(\widehat{X}^{(i)}\right)-\mathbb{E}\left[\Phi\left(\widehat{X}^{(i)}\right)\right]\right)\right), \\
\text { and } \mu_{\beta}^{\prime} & =\frac{1}{N} \sum_{i=1}^{N}\left(\Phi\left(X^{(i)}\right)-\beta^{(i)}\left(\Phi\left(\widehat{X}^{(i)}\right)-\mathbb{E}\left[\Phi\left(\widehat{X}^{(i)}\right)\right]\right)\right),
\end{aligned}
$$

where $\beta^{(i)}$ is constructed from $X^{(1)}, \ldots, X^{(i)}$ to approximate $\beta_{0}$ in (2).

The results are summarized in Tables 1, 2 and 3 . The mean of the estimator is denoted by $\mu$ (in all the case, the relative error between the different empirical estimator is very small), which is then the price of the option.

The efficiency of a variance reduction technique shall take the computation time into account. In Tables 1,2 and $3, T$ is the time spent to compute the empirical mean $\mu$ without the control variate, while $T^{\prime}$ is the time spent to compute the empirical mean $\mu$ with the control variate (time $\theta_{\text {simul }}$ per simulation). With our control variate, the extra computational time is due to (a) the initialization of the algorithm (time $T_{\text {init }}$ ), which depends then on the number of points of quantization, and (b) the research of the nearest neighbor of the Gaussian coefficients among the quantized points (time $\theta_{\text {search }}$ per simulation). For researching the nearest neighbor of the vector, we have used a methods called $k d$-tree (see for example [3]) which appears to be efficient, while for a scalar, we used a dichotomy search.

The method have been applied to several call options, for three values of $K: K=0.5$ (In the money, ITM), $K=1.0$ (At the money, ATM) 
and $K=1.3$ (Out of the money, OTM). The options are

European: $\Phi(X)=\left(X_{T}-K\right)_{+}$,

Asian: $\Phi(X)=\left(\frac{1}{T} \int_{0}^{T} X_{s} \mathrm{~d} s-K\right)_{+}$,

Asian binary: $\Phi(X)=\mathbf{1}_{\frac{1}{T} \int_{0}^{T} X_{s} \mathrm{~d} s>K}$,

Ratchet: $F_{0}=0, F_{i}=\left(K+F_{i-1}+\rho_{i}\right)_{+}$and $\rho_{i}=\frac{X_{t_{i}}-X_{t_{i-1}}}{\left(t_{i}-t_{i-1}\right) X_{t_{i}}}$,

Lookback fixed: $\Phi(X)=X_{T}-\min _{t \in[0, T]} X_{t}$,

Lookback floating: $\Phi(X)=\left(\max _{t \in[0, T]} X_{t}-K\right)_{+}$,

$$
\text { Cliquet: } \max \left\{F_{0}, \min \left\{C_{0}, \sum_{i=1}^{N} \max \left\{F_{i}, \min \left\{C_{i}, R_{i}\right\}\right\}\right\}\right.
$$

where the floors $F_{i}=0$ and the caps $C_{i}=K$, and $R_{i}=X_{t_{i}}-X_{t_{i-1}}$. For the cliquet and ratchet options, $t_{i}=i T / 12$.

Thus, with $N$ simulations and $Q$ quantized path, the ratio of the times $T^{\prime} / T$ is given by

$$
\frac{T^{\prime}}{T}=\frac{T_{\text {init }}(Q)+N \theta_{\text {search }}+N \theta_{\text {simul }}}{N \theta_{\text {simul }}}
$$

Let us note that the initialization phase implies the resolution of $Q$ ordinary differential equations, so that $T_{\text {init }} \approx Q \theta_{\text {simul }}$.

Thus, in Tables 1, 2 and 3, we give the efficiency ratio for 100,000 and 1,000,000 simulations. We call the efficiency ratio the quantities

$$
e=\frac{T}{T^{\prime}} \times \frac{v}{v^{\prime}} \text { and } e_{\beta}=\frac{T}{T^{\prime}} \times \frac{v}{v_{\beta}^{\prime}} .
$$

When these quantities are close to 1 , then the method provides no gain. It is then simpler to increase the size of the sample without any correction to obtain the same result. When these quantities are greater than 1, then for a given precision, it corresponds to the gain factor in execution time. It these quantities are smaller than 1, then the methods shall not be used.

For most of the simulations, the variance reduction technique is efficient or very efficient: The efficiency ratios above 5 have been written in bold. For very small values of prices, the methods may be inefficient (the figures are then emphasized). In this case, using the estimator $\mu_{\beta}^{\prime}$ improves the quality of the estimator, but does not provides significant gains with respect to $\mu$. Most the of time, using $\mu_{\beta}^{\prime}$ instead of $\mu^{\prime}$ does not improve significatively the results. In addition, the method may also be inefficient when the payoff is discontinuous, as one may expect. 


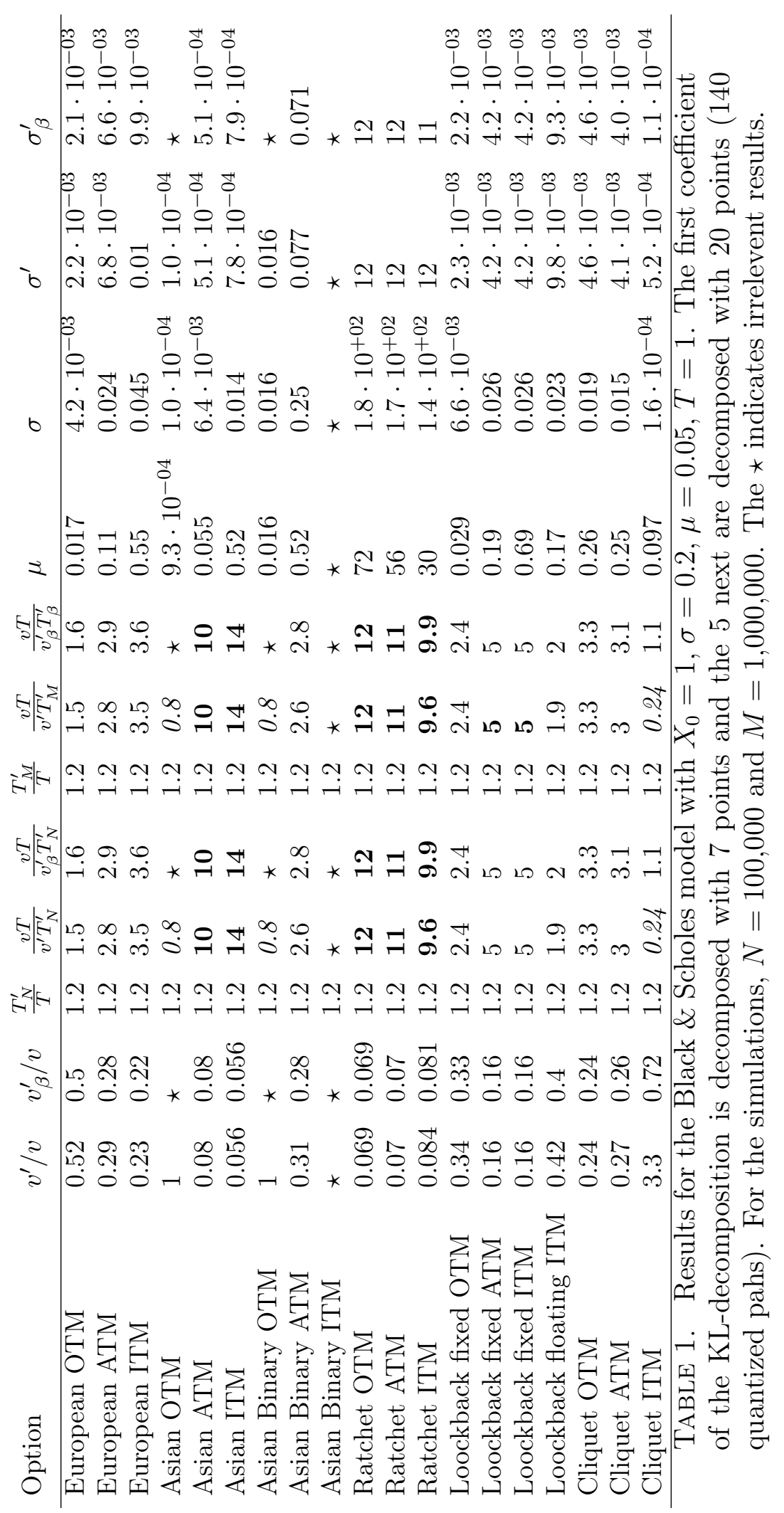




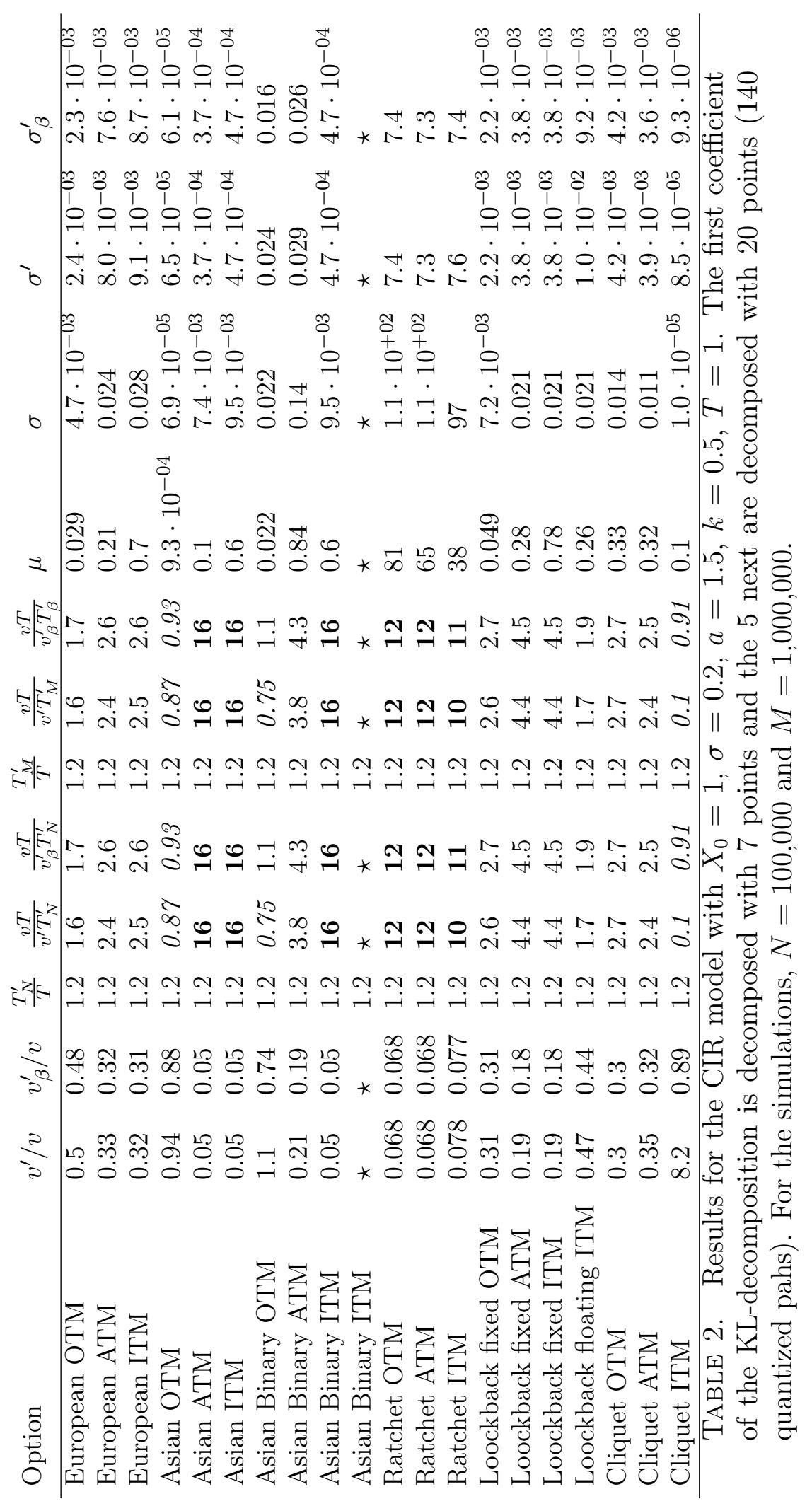




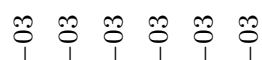

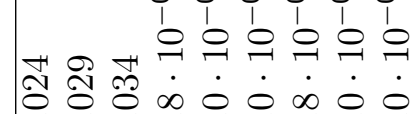

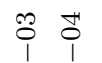

药

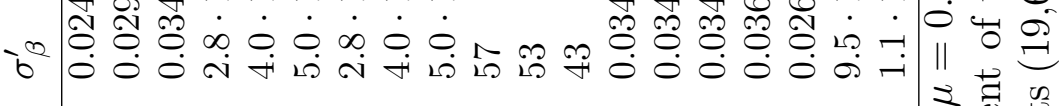

$\approx \approx \% \approx \approx$

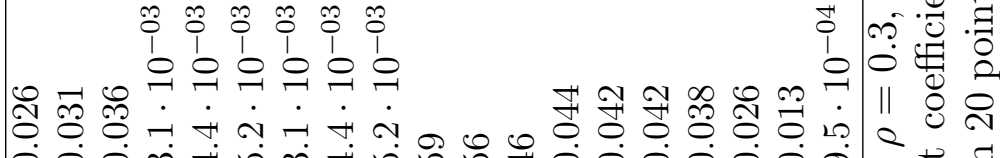

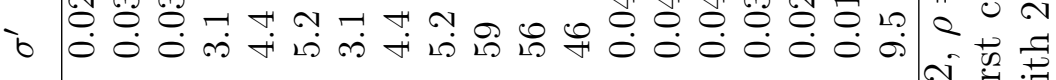

ชิ

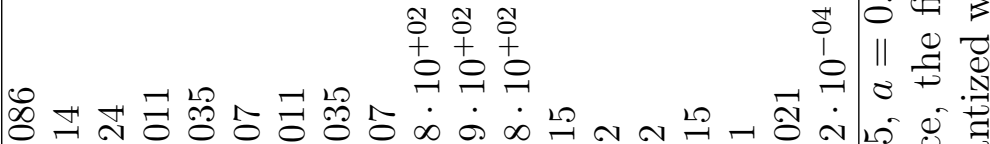

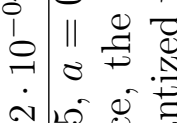

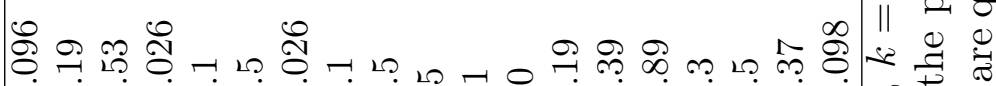

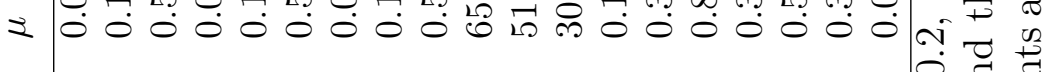

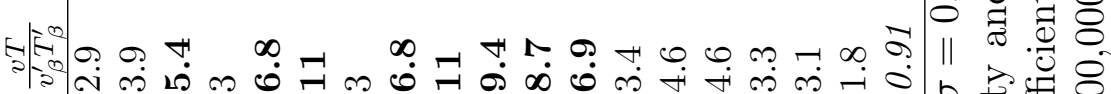

A

Ia $\quad$ i

Eस

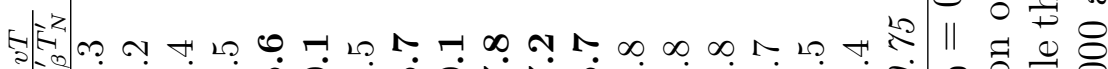

ح/

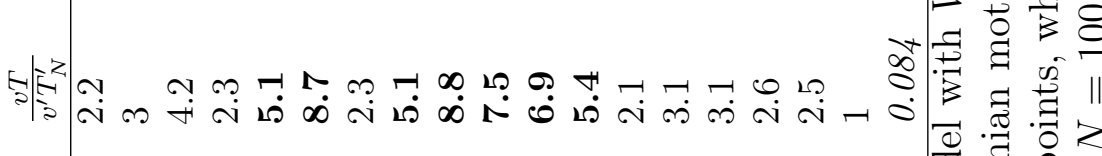

Eस|ה

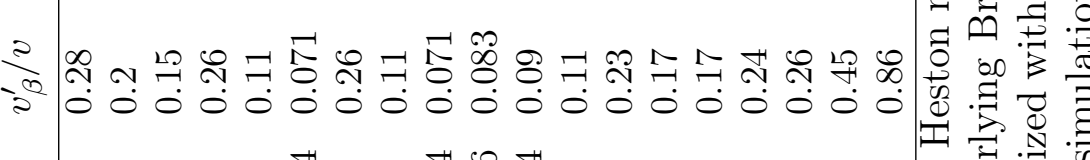

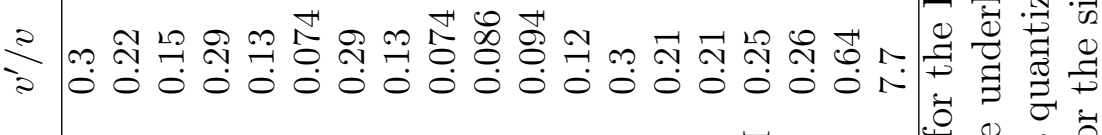

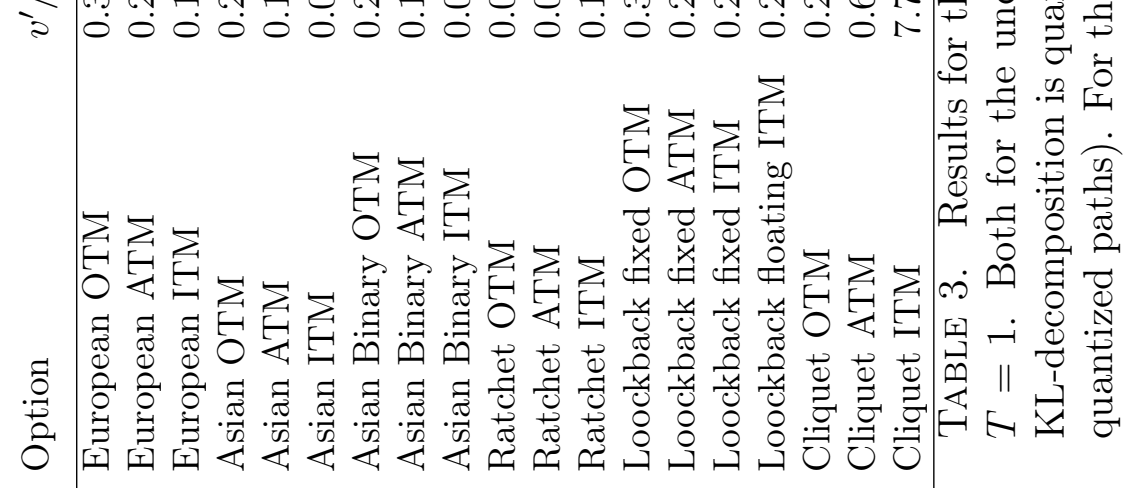




\section{Conclusion}

We have presented a new variance reduction technique which belongs to the class of hybrid quantization-Monte Carlo method related to the simulation of SDE.

This method relies on functional quantization and may be combined with other variance reduction techniques applied to the Brownian motion, as the payoff is changed.

Numerical tests show the robustness and the efficiency of the method, with good gain factors, up to order 10, even for complex payoffs. However, this method is not suitable for SDEs with a large number of underlying Brownian motions - as the number of quantized paths growth very quickly - and to estimate small quantities or prices.

As the end-user is free to fix a lot of parameters (number of quantization points, way to decompose the vectors, ...), the numerical results presented here may be improved by tuning the parameters to get the best balance between a small ratio of variances and the extra computational cost. Note that however, this tuning should be model dependent.

\section{Appendix A. The solution of the SDE as a FunCtional of} THE BROWNIAN MOTION

Let us consider the SDE

$$
X_{t}=x+\int_{0}^{t} \sigma\left(X_{s}\right) \mathrm{d} B_{s}+\int_{0}^{t} b\left(X_{s}\right) \mathrm{d} s
$$

where $B$ is a $d$-dimensional Brownian motion, $X$ takes its values in $\mathbb{R}^{d}$, $\sigma$ is a function of class $\mathcal{C}^{2}$ on $\mathbb{R}^{d}$ that takes its values in the space of $d \times m$-matrix and $b$ is a $\mathcal{C}^{1}$ function from $\mathbb{R}^{d}$ to $\mathbb{R}^{d}$. We assume that both $b$ and $\sigma$ have at most a linear growth, so that (5) has a unique strong solution [12].

We may rewrite (5) as a Stratonovich SDE

$$
X_{t}=x+\int_{0}^{t} \sigma\left(X_{s}\right) \circ \mathrm{d} B_{s}+\int_{0}^{t} c\left(X_{s}\right) \mathrm{d} s
$$

with

$$
c_{i}(x)=b_{i}(x)-\sum_{j, k=1}^{d} \frac{1}{2} \frac{\partial \sigma_{i, k}(x)}{\partial x_{j}} \sigma_{j, k}(x) .
$$

For a piecewise smooth path $U$, one can also consider the ordinary differential equation (ODE)

$$
Y_{t}=x+\int_{0}^{t} \sigma\left(Y_{s}\right) \mathrm{d} U_{s}+\int_{0}^{t} c\left(Y_{s}\right) \mathrm{d} s .
$$


A.1. The one dimensional case: the Doss-Sussman transform. Let us defined by $u(\cdot ; y)$ the family of solutions

$$
\frac{\partial u(x, y)}{\partial x}=\sigma(u(x, y)) \text { with } u(0, y)=y
$$

and

$$
\frac{\mathrm{d} Y_{t}}{\mathrm{~d} t}=f\left(W_{t}, Y_{t}\right) \text { with } Y_{0}=x \text { with } f(x, y)=\frac{c(u(x, y))}{\partial_{y} u(x, y)}
$$

Then the results from H. Doss [7] and H. Sussmann [28] (see also [12, p. 295] or [24]) assert the solution to (6) (or equivalently (5)) is given by $X_{t}=u\left(W_{t}, Y_{t}\right)$. In addition, if $V_{t}$ is solution to $V_{t}^{\prime}=f\left(U_{t}, V_{t}\right)$ where $U$ is a piecewise smooth path, then the solution $Y$ to (7) is given by $Y_{t}=u\left(U_{t}, V_{t}\right)$. Let us denote by $\Theta$ the map that transforms $U \in \mathcal{C}_{\mathrm{p}}^{\infty}([0, T] ; \mathbb{R})$ to $Y$ by this way. Then $\Theta$ may be extended to the space of continuous functions and is locally Lipschitz continuous with respect to the uniform norm. In addition, $X=\Theta(B)$ almost surely.

A.2. The multi-dimensional case: an approach via rough paths. If the diffusion $X$ and the underlying process $B$ take their values in $\mathbb{R}^{n}$, the situation is more complicated, as one cannot extend in a continuous and canonical way an ordinary ODE driven by a smooth path to a differential equation driven by some irregular path.

From the Yamada-Watanabe theorem [12], one knows that there exists some measurable function $\Psi(\cdot ; x)$ such that $X=\Psi(B ; x)$ for the Brownian motion $B$ when $X_{0}=x$.

The rough path theory, developed from the 90 ' by T. Lyons and his co-authors, allows one to be more precise $[13,14,19,20]$, and to define a notion of ordinary differential equations driven by an irregular path in a continuous way.

Indeed, let us consider the set of trajectories that take their values in $T\left(\mathbb{R}^{n}\right)=\mathbb{R}^{n} \oplus \mathcal{M}_{n \times n}(\mathbb{R})$, where $\mathcal{M}_{n \times n}(\mathbb{R})$ is the space of $n \times n$ matrices. Any element in $\mathcal{M}_{n \times n}(\mathbb{R})$ will be identified with an element of the tensor space $\mathbb{R}^{n} \otimes \mathbb{R}^{n}$.

For two points $x$ and $y$ in $T\left(\mathbb{R}^{n}\right)$, we consider the jauge $\delta(x, y)=$ $\max \left\{\delta_{1}(x), \sqrt{\delta_{2}(x)}\right\}$ with

$$
\begin{aligned}
& \delta_{1}(x, y)=\left|\pi_{\mathbb{R}^{n}}(x)-\pi_{\mathbb{R}^{n}}(y)\right|, \\
& \delta_{2}(x, y)=\left|\pi_{\mathcal{M}_{n \times n}(\mathbb{R})}(y)-\pi_{\mathcal{M}_{n \times n}(\mathbb{R})}(y)-\alpha(y-x, y)\right|,
\end{aligned}
$$

where $\pi_{\mathrm{X}}$ is the linear projection on $\mathrm{X}$, and $\alpha(x, y)$ is the matrix $\left(x_{i} y_{j}\right)_{i, j=1, \ldots, n}$ with $\pi_{\mathbb{R}^{n}}(x)=\left(x_{1}, \ldots, x_{d}\right)$. 
For $p \in[2,3)$, denote by $\mathcal{R}_{p}\left(\mathbb{R}^{n}\right)$ the set of paths $\mathbf{X}$ with values in $T\left(\mathbb{R}^{n}\right)$ such that

$$
\begin{array}{r}
\|\mathrm{X}\|_{p}=\sup _{t \in[0, T]} \delta\left(0, \mathrm{X}_{t}\right)+\sup _{\text {finite partitions }\left\{t_{i}\right\} \text { of }[0, T]}\left(\sum_{i=1}^{+\infty} \delta_{1}\left(\mathrm{X}_{t_{i}}, \mathrm{X}_{t_{i+1}}\right)^{p}\right)^{1 / p} \\
+\sup _{\text {finite partitions }\left\{t_{i}\right\} \text { of }[0, T]}\left(\sum_{i=1}^{+\infty} \delta_{2}\left(\mathrm{X}_{t_{i}}, \mathrm{X}_{t_{i+1}}\right)^{p / 2}\right)^{2 / p}
\end{array}
$$

is finite. This set is called the set of rough paths of finite p-variation. One of the main results of $\mathrm{T}$. Lyons is that for a set of functions $f_{1}, \ldots, f_{d}$ of class $\mathcal{C}_{\mathrm{b}}\left(\mathbb{R}^{n} ; \mathbb{R}^{n}\right)$ and $x \in \mathbb{R}^{n}$, it is possible to define a map $\mathfrak{I}$ from $\mathcal{R}^{p}\left(\mathbb{R}^{n}\right)$ to $\mathcal{R}^{p}\left(\mathbb{R}^{n}\right)$ which is (locally Lipschitz [15]) continuous with respect to the topology generated by $\|\cdot\|_{p}$, and such that, when restricted to the set $\mathrm{X}$ such that $\pi_{\mathbb{R}^{n}}(\mathrm{X})=X$ for a piecewise smooth path $X$ and $\pi_{\mathcal{M}_{n \times n}(\mathbb{R})}\left(\mathrm{X}_{t}\right)=\int_{0}^{t}\left(X_{s}-X_{0}\right) \otimes \mathrm{d} X_{s}$, then $\mathrm{Y}=\mathfrak{I}(\mathrm{X})$ is such that $\pi_{\mathcal{M}_{n \times n}(\mathbb{R})}\left(\mathrm{Y}_{t}\right)=\int_{0}^{t}\left(Y_{s}-Y_{0}\right) \otimes \mathrm{d} Y_{s}$ where $\pi_{\mathbb{R}^{n}}(\mathrm{Y})=Y$ is given by

$$
Y_{t}=y+\int_{0}^{t} f\left(Y_{s}\right) \mathrm{d} X_{s}
$$

Remark 3. Time-inhomogeneous SDEs can be treated as well, but we deal here only with homogeneous SDEs for the sake of simplicity. If $\mathbf{X}$ is a rough path in $\mathcal{R}^{p}\left(\mathbb{R}^{n}\right)$ with $p \in[2,3)$, then one may consider the map $\Xi: \mathcal{R}^{p}\left(\mathbb{R}^{n}\right) \rightarrow \mathcal{R}^{p}\left(\mathbb{R}^{n+1}\right)$ such that $\mathrm{Y}=\Xi(\mathrm{X})$ is defined by

$$
\mathrm{Y}_{t}=\mathrm{Y}_{t}+\int_{0}^{t}\left(Y_{s}-Y_{0}\right) \otimes e_{n+1} d s+\int_{0}^{t} s e_{n+1} \otimes \mathrm{d} Y_{s}+\frac{t^{2}}{2} e_{n+1} \otimes e_{n+1},
$$

with $Y_{t}=\pi_{\mathbb{R}^{n}}\left(\mathrm{Y}_{t}\right)$, and $\left\{e_{1}, \ldots, e_{n+1}\right\}$ the canonical basis of $\mathbb{R}^{n+1}$ where the $n+1$-component is the component of the time. This map $\Xi$ is continuous from $\left(\mathcal{R}^{p}\left(\mathbb{R}^{n}\right),\|\cdot\|_{p}\right)$ to $\left(\mathcal{R}^{p}\left(\mathbb{R}^{n+1}\right),\|\cdot\|_{p}\right)$. One can then consider a time-inhomogeneous SDE driven by $X$ as a time-homogeneous SDE driven by $Y$. Using the theory of $(p, q)$-rough paths [17], it is possible to relax the regularity assumptions on the coefficients involving the time (as a driver or as a variable).

A Brownian motion $B$ belongs to $\mathcal{R}^{p}\left(\mathbb{R}^{n}\right)$ for any $p \in[2,3)$ (see for example $[4,27])$. In addition the solution $X$ to $(6)$ is the projection on $\mathbb{R}^{n}$ of $\mathrm{X}=\mathfrak{I}(\mathrm{B}) \in \mathcal{R}^{p}\left(\mathbb{R}^{n}\right)$ with

$$
\mathrm{B}_{t}=B_{t}+\int_{0}^{t} B_{s} \otimes \circ \mathrm{d} B_{s}
$$

where $\left(f_{1}, \ldots, f_{m+1}\right)=\left(\sigma_{\cdot, 1}, \ldots, \sigma_{\cdot, m}, c\right)$, provided that $\sigma$ and $c$ are smooth enough, for example $\sigma \in \mathcal{C}^{3}$ and $c \in \mathcal{C}^{2}$.

One can indeed recover the classical Wong-Zakai theorem. If $\left(B^{n}\right)_{n \in \mathbb{N}}$ is a family of piecewise linear approximations of the Brownian motion 
along the dyadic partitions, and $\mathrm{B}^{n}=\Lambda\left(B^{n}\right)$ with $\Lambda: X \in \mathcal{C}^{1}\left([0, T] ; \mathbb{R}^{n}\right) \rightarrow X+\int_{0}^{t}\left(X_{s}-X_{0}\right) \otimes \mathrm{d} X_{s} \in \mathcal{R}^{1}\left(\mathbb{R}^{n}\right) \subset \mathcal{R}^{p}\left(\mathbb{R}^{n}\right)$, then $\mathrm{B}^{n}$ converges to $B$ in $\left(\mathcal{R}^{p}\left(\mathbb{R}^{n}\right),\|\cdot\|_{p}\right)[4,27]$. One has to take care that this is not true for any choice of family of approximations of the Brownian motion (see for example $[14,16]$ for some explicit counter-examples). Indeed, when $n>1, \Lambda$ is not continuous, even from $\mathcal{C}^{1}\left([0, T] ; \mathbb{R}^{n}\right)$ to $\mathcal{R}^{p}\left(\mathbb{R}^{n}\right)$ with respect to the uniform norm.

\section{A.3. Convergence of the Karhunen-Loève expansion as a rough} path. Let $\widetilde{B}^{m}$ be the truncated expansion of the multi-dimensional Brownian on the Karhunen-Loève decomposition:

$$
\widetilde{B}_{t}^{m}=\left[\begin{array}{c}
\sum_{i=1}^{m} \sqrt{\lambda_{i}} \xi_{1, i} f_{i}(t) \\
\vdots \\
\sum_{i=1}^{m} \sqrt{\lambda_{i}} \xi_{d, i} f_{i}(t)
\end{array}\right], t \in[0, T],
$$

with $\boldsymbol{\xi}=\left(\xi_{j, i}\right)_{j=1, \ldots, d, i=1, \ldots, m}$ is a $d \times m$-matrix with independent coefficients that are realization of normal distributions with mean 0 and variance 1 .

Let $\widetilde{\mathrm{B}}^{m}=\Lambda\left(\widetilde{B}^{m}\right)$. In [5], it is proved that $\widetilde{\mathrm{B}}^{m}$ converges almost surely to $\mathrm{B}$ in $\left(\mathcal{R}^{p}\left(\mathbb{R}^{d}\right),\|\cdot\|_{p}\right)$ as $m \rightarrow \infty$.

Let $(\boldsymbol{\xi}(r))_{r \in \mathbb{N}}$ be a sequence of coefficients in $\mathbb{R}^{m}$ that converges to $\boldsymbol{\xi}$ in $\mathbb{R}^{m}$, and set

$$
\widetilde{B}_{t}^{m}(r)=\sum_{j=1}^{d}\left(\sum_{i=1}^{m} \sqrt{\lambda_{i}} \xi_{k, i}(r) f_{i}(t)\right) e_{j}, t \in[0, T],
$$

where $\left(e_{1}, \ldots, e_{d}\right)$ is the canonical basis of $\mathbb{R}^{d}$. Then

$$
\begin{aligned}
& \widetilde{\mathrm{B}}^{m}(r):=\Lambda\left(\widetilde{B}^{m}(r)\right)=\widetilde{B}_{t}^{m}(r) \\
& \quad+\sum_{k, \ell=1}^{d}\left(\sum_{i, j=1}^{m} \sqrt{\lambda_{i} \lambda_{j}} \xi_{k, i}(r) \xi_{\ell, j}(r) \int_{0}^{t}\left(f_{i}(s)-f_{i}(0)\right) \mathrm{d} f_{j}(s)\right) e_{k} \otimes e_{\ell} .
\end{aligned}
$$

Hence, when $m$ is fixed, it is clear that $\left(\left\|\widetilde{\mathrm{B}}^{m}(r)\right\|_{p}\right)_{r \in \mathbb{N}}$ remains bounded, as all the sequences $\left(\xi_{i, j}(r)\right)_{n \in \mathbb{N}}$ are bounded. Thus, from an adaptation of the Ascoli-Arzelà theorem (see [13]), we get that $\widetilde{\mathrm{B}}^{m}(r)$ converges in $\mathcal{R}^{q}\left(\mathbb{R}^{d}\right)$ to $\mathrm{B}$ as $r \rightarrow \infty$ for any $q>p$.

Thus, thanks to the continuity of the Itô map, the solution to (7) with $U=\widetilde{B}^{m}(n)$ converges almost surely in $p$-variation (and thus uniformly) to the solution to $(6)$.

On the other hand, G. Pagès and A. Sellami have proved in [26] that if $\left(\widehat{B}^{N}\right)_{N \in \mathbb{N}}$ is a sequence of stationary quadratic functional quantizers of the Brownian motion, then $\Lambda\left(\widehat{B}^{N}\right)$ is a stationary quadratic 
functional quantizer of B. They have also studied the properties of convergence.

\section{A.4. Is our control variate a good approximation of the diffu-}

sion? With our algorithm, we construct an family of paths $\left\{\widehat{B}^{(i)}\right\}_{i=1, \ldots, M}$ and a rule, through the Karhunen-Loève decomposition, to associate one of the $\widehat{B}^{(i(B))}$ to any realization of the Brownian motion $B$, where $i(B)$ is the index such that $\widehat{\boldsymbol{\alpha}}^{(i)}=\pi(\boldsymbol{\alpha})$ in our algorithm. Using $\widehat{B}^{(i(B))}$ as a control variate, the variance of our estimator is given by $N^{-1} \operatorname{Var}\left(\Phi(B)-\Phi\left(\widehat{B}^{(i(B))}\right)\right)$, while the variance of the standard Monte Carlo estimator is $N^{-1} \operatorname{Var}(\Phi(B))$, when $N$ samples are drawn. Thus, the quality of the estimator depends crucially on the fact that $\widehat{B}^{i(B)}$ is close to $B$ when $\Phi$ is continuous.

With the previous results, we have seen that it is sufficient to consider how close to $B$ is $\widehat{B}^{i(B)}$.

The distance between $B$ and $\widehat{B}^{i(B)}$ is due to three factors: (i) the truncation number $m$; (ii) the quantization error on the coefficients $\boldsymbol{\xi}$; (iii) the difference between the exact Karhunen-Loève decomposition and the one given by the least squares approximation, but which can be neglected in practice for good choices of orthonormal basis of $\mathrm{L}^{2}([0, T] ; \mathbb{R})$ if one chooses to quantize normal distribution with covariance matrix equal to Id.

The distance between a Brownian path and a path constructed using the quantization of the Gaussian coefficient in the truncated KarhunenLoève decomposition has been studied by G. Pagès and J. Printems in [24] and it covers thus (i) and (ii). Here, the distance is increased by the fact that we use a decomposition of Karhunen-Loève type whose coefficients have a covariance matrix that may be different from Id. We now study this distance.

We consider only the case of a one-dimensional Brownian motion, but the multi-dimensional case may be treated as well using the results of Section A.3.

If the number of points of quantification converges to $+\infty$, then the distance between the quantized point $\pi(\boldsymbol{\alpha})$ and $\boldsymbol{\alpha}$ converges to 0 , and then $\widehat{B}_{t}^{(i(B))}$ converges to

$$
\widetilde{B}_{t}^{m}(d):=\sum_{j=1}^{m} \xi_{j}(d) \sqrt{\lambda_{j}} f_{j}(t) \text { with } \boldsymbol{\xi}(d)=\Gamma \boldsymbol{\alpha} .
$$

Let us denote by $\Gamma(d)$ a symmetric $d \times d$-matrix such that $\Gamma(d) \Gamma(d)^{T}$ is the covariance matrix of the vector $\boldsymbol{\xi}(d)$ obtained by solving a least squares problem

$$
\boldsymbol{\xi}(d)=\operatorname{argmin}_{\boldsymbol{\zeta}(d) \in \mathbb{R}^{m}} \sum_{i=1}^{d}\left(B_{t_{i}}-\sum_{j=1}^{m} \zeta_{j}(d) \sqrt{\lambda_{j}} f_{j}\left(t_{i}\right)\right)^{2}
$$


Thus, this matrix depends on the number of coefficients $m$ as well as the times $\left(t_{1}, \ldots, t_{d}\right)$ of the marginals of the Brownian motion. We now consider that $m$ is fixed and that $d$ increases to $+\infty$.

Lemma 1. If $t_{i}^{d}=i T / d$ for $i=1, \ldots, d$, then the symmetric $d \times d$ matrix $\Gamma(d)$ with $\Gamma(d)^{2}=\operatorname{Cov}(\boldsymbol{\xi}(d))$ converges to the identity as $d \rightarrow \infty$ and $\widetilde{B}_{t}^{m}(d)$ converges almost surely uniformly to the Karhunen-Loève decomposition $\widetilde{B}^{m}$ of $B$ truncated at order $m$.

Proof. Let $M(d)$ be the matrix defined by $M_{i, j}(d)=\sqrt{\lambda_{j}} f_{j}\left(t_{i}^{d}\right)$ for $t_{i}^{d}=i T / d$, as in Section 2.2, and set $\boldsymbol{\beta}(d)=\left(B_{t_{1}^{d}}, \ldots, B_{t_{d}^{d}}\right)$. Thus as $d \rightarrow \infty$,

$$
\frac{T}{d} M(d) \boldsymbol{\beta}(d)=\left[\begin{array}{c}
\frac{T}{d} \sum_{i=1}^{d} \sqrt{\lambda_{1}} f_{1}\left(t_{i}^{d}\right) B_{t_{i}^{d}} \\
\vdots \\
\frac{T}{d} \sum_{i=1}^{d} \sqrt{\lambda_{m}} f_{m}\left(t_{i}^{d}\right) B_{t_{i}^{d}}
\end{array}\right] \stackrel{d \rightarrow \infty}{\longrightarrow}\left[\begin{array}{c}
\sqrt{\lambda_{1}} \int_{0}^{T} f_{1}(s) B(s) \mathrm{d} s \\
\vdots \\
\sqrt{\lambda_{m}} \int_{0}^{T} f_{m}(s) B(s) \mathrm{d} s
\end{array}\right]
$$

Let $\widetilde{B}_{t}^{m}:=\sum_{j=1}^{m} \xi_{j} \sqrt{\lambda_{j}} f_{j}(t), t \in[0, T]$, be the truncated KarhunenLoève decomposition of $B$. Since $\left\{f_{i}\right\}_{i \geq 0}$ is an orthonormal basis of $\mathrm{L}^{2}([0, T])$, one gets easily that $\lambda_{i} \xi_{i}=\sqrt{\lambda_{i}} \int_{0}^{T} f_{i}(s) B(s) d s$ for $i=$ $1, \ldots, m$, and then that $T d^{-1} M(d) \boldsymbol{\beta}(d)$ converges to the vector $L \boldsymbol{\xi}$ with $\boldsymbol{\xi}=\left(\xi_{1}, \ldots, \xi_{m}\right)$, where $L$ is the diagonal matrix with $\left(\lambda_{1}, \ldots, \lambda_{m}\right)$ as diagonal elements. On the other hand, one easily gets from the same argument that

$$
\frac{T}{d} M(d) M(d)^{T} \stackrel{d \rightarrow \infty}{\longrightarrow}\left(\sqrt{\lambda_{i} \lambda_{j}} \int_{0}^{T} f_{i}(s) f_{j}(s) d s\right)_{i, j=1, \ldots, d}=L .
$$

Since the $\lambda_{i}$ are positive, $Q(d)=T d^{-1} M(d) M(d)^{T}$ is invertible for $d$ large enough and $Q(d)^{-1}$ converges to $L^{-1}$. It follows that

$$
\boldsymbol{\xi}(d)=Q(d)^{-1} T d^{-1} M(d) \boldsymbol{\beta}(d) \underset{d \rightarrow \infty}{\longrightarrow} L^{-1} L \boldsymbol{\xi}=\boldsymbol{\xi} .
$$

The uniform convergence of $\widetilde{B}^{m}$ to $B$ follows from the convergence of $\boldsymbol{\xi}(d)$ to the coefficients $\boldsymbol{\xi}$ of the Karhunen-Loève decomposition of $B$, and the convergence of $\Gamma(d)$ to Id follows from the fact that $\operatorname{Cov}(\boldsymbol{\xi}(d))$ converges to $\operatorname{Cov}(\beta)=\mathrm{Id}$ and then $\Gamma(d)$ also converges to Id (see [29] for example).

Remark 4. From a practical point of view, numerical experiments show that the covariance matrix $\operatorname{Cov}(\boldsymbol{\xi}(d))$ is already close to Id even for small values of $d$ when the basis of Remark 1 is used. Thus, one can take for $d$ values of order 10 when $m \leq 10$ to get from the least squares approach a good approximation of the truncated KarhunenLoève decomposition of $B$. 
Acknowlegement. The authors are indebted to Aymen Bergaoui, Madalina Deaconu, Zied Ghazai, Samuel Herrmann, Christophe Michel, Gilles Pagès, Yiqing Wang for interesting discussions about this method or problems related to quantization.

\section{REFERENCES}

[1] V. Bally, G. Pagès, and J. Printems, A quantization tree method for pricing and hedging multidimensional American options, Math. Finance 15 (2005), no. 1, 119-168.

[2] O. Bardou, S. Bouthemy, and G. Pagès, Optimal quantization for the pricing of swing options (2007), available at arxiv:0705.2110.

[3] J.L. Bently, Multidimensional divide-and-conquer, Communications of the ACM 23 (1980), no. 4, 214-229.

[4] L. Coutin and A. Lejay, Semi-martingales and rough paths theory, Electron. J. Probab. 10 (2005), no. 23, 761-785.

[5] L. Coutin and N. Victoir, Enhanced Gaussian Processes and Applications, ESAIM Probab. Stat., posted on 2008, DOI 10.1051/ps:2008007, (to appear in print).

[6] F. Delarue and S. Menozzi, An interpolated stochastic algorithm for quasilinear PDEs, Math. Comp. 77 (2008), no. 261, 125-158.

[7] H. Doss, Liens entre équations différentielles stochastiques et ordinaires, Ann. Inst. H. Poincaré Sect. B (N.S.) 13 (1977), no. 2, 99-125.

[8] G. S. Fishman, Monte Carlo, Springer Series in Operations Research, SpringerVerlag, New York, 1996. Concepts, algorithms, and applications.

[9] P. Friz and N. Victoir, Differential Equations Driven by Gaussian Signals II (2007), available at arxiv:0711.0668. Preprint.

[10] P. Glasserman, Monte Carlo methods in financial engineering, Applications of Mathematics (New York), vol. 53, Springer-Verlag, New York, 2004. Stochastic Modelling and Applied Probability.

[11] S. Graf and H. Luschgy, Foundations of quantization for probability distributions, Lecture Notes in Mathematics, vol. 1730, Springer-Verlag, Berlin, 2000.

[12] I. Karatzas and S. E. Shreve, Brownian motion and stochastic calculus, 2nd ed., Graduate Texts in Mathematics, vol. 113, Springer-Verlag, New York, 1991.

[13] A. Lejay, An introduction to rough paths, Séminaire de probabilités XXXVII, Lecture Notes in Mathematics, vol. 1832, Springer-Verlag, 2003, pp. 1-59.

[14] _ Yet another introduction to rough paths (2009). To appear in Séminaire de probabilités, Lecture Notes in Mathematics, Springer-Verlag.

[15] _ On rough differential equations, Electron. J. Probab. 14 (2009), no. 12, 341-364.

[16] A. Lejay and T. Lyons, On the importance of the Lévy area for systems controlled by converging stochastic processes. Application to homogenization, New Trends in Potential Theory, Conference Proceedings, Bucharest, September 2002 and 2003 (D. Bakry, L. Beznea, Gh. Bucur, and M. Röckner, eds.), The Theta Foundation, 2006, pp. 63-84.

[17] A. Lejay and N. Victoir, On $(p, q)$-rough paths, J. Differential Equations 225 (2006), no. 1, 103-133, DOI 10.1016/j.jde.2006.01.018.

[18] H. Luschgy and G. Pagès, Functional quantization of a class of Brownian diffusions: a constructive approach, Stochastic Process. Appl. 116 (2006), no. 2, 310-336.

[19] Terry J. Lyons, Differential equations driven by rough signals, Rev. Mat. Iberoamericana 14 (1998), no. 2, 215-310. 
[20] Terry Lyons and Zhongmin Qian, System control and rough paths, Oxford Mathematical Monographs, Oxford University Press, Oxford, 2002. Oxford Science Publications.

[21] G. Pagès, H. Pham, and J. Printems, Optimal quantization methods and applications to numerical problems in finance, Handbook of computational and numerical methods in finance, Birkhäuser Boston, 2004, pp. 253-297.

[22] G. Pagès and J. Printems, Web site devoted to vector and functional optimal quantization: www. quantize.maths-fi.com, 2005.

[23] G. Pagès and J. Printems, Optimal quadratic quantization for numerics: the Gaussian case, Monte Carlo Methods Appl. 9 (2003), no. 2, 135-165.

[24]_, Functional quantization for numerics with an application to option pricing, Monte Carlo Methods Appl. 11 (2005), no. 4, 407-446.

[25] _ Optimal quantization for Finance: from random vectors to stochastic processes (2008). Preprint, Université Paris 6.

[26] G. Pagès and A. Sellami, Convergence of multi-dimensional quantized SDE's (2008), available at arxiv:0801.0726.

[27] E.-M. Sipiläinen, A pathwise view of solutions of stochastic differential equations, Ph.D. thesis, University of Edinburgh, 1993.

[28] H.J. Sussmann, On the gap between deterministic and stochastic ordinary differential equations, Ann. Probability 6 (1978), no. 1, 19-41.

[29] D. W. Stroock, Lectures on stochastic analysis: diffusion theory, London Mathematical Society Student Texts, vol. 6, Cambridge University Press, Cambridge, 1987.

[30] R. Szechtman, Control variate techniques for Monte Carlo simulation, Proceedings of the 2003 Winter Simulation Conference (S. Chick, P.J. Sánchez, D. Ferrin, and D.J. Morrice, eds.), 2003, available at www.informs-cs.org/ wsc03papers/017.pdf.

A. Lejay, Projet Tosca, Institut Elie Cartan Nancy, Nancy-Université, CNRS, INRIA, Boulevard des Aiguillettes B.P. 239 F-54506 VandøeuVRE LÈS NANCY CEDEX, FRANCE.

E-mail address: Antoine.Lejay@iecn.u-nancy.fr

$U R L:$ http://www.iecn.u-nancy.fr/ lejay

V. Reutenauer, Calyon, Fim, Interest Rates and Hybrid Quantitative Research, 9 Quai du Président Paul Doumer, 92920 Paris-La DÉFENSE CEDEX, FRANCE

E-mail address: Victor.Reutenauer@calyon.com 